\title{
A survey of the working status of family medicine physicians in clinics and hospitals in Korea
}

Hyun-Young Shin ( $\square$ shydeborah@gmail.com )

Hanyang University

Research article

Keywords: continuity of care, family medicine, practice management, primary care

Posted Date: April 1st, 2020

DOI: https://doi.org/10.21203/rs.2.15645/v3

License: (c) (i) This work is licensed under a Creative Commons Attribution 4.0 International License.

Read Full License

Version of Record: A version of this preprint was published at BMC Family Practice on May 8th, 2020. See the published version at https://doi.org/10.1186/s12875-020-01154-5. 


\section{Abstract}

Background In the approximately 35 years since family medicine was established in South Korea, family medicine physicians have sought to expand their expertise to cover clinical fields beyond primary medicine. This study examines their working status and compares the working conditions of family medicine physicians in clinics and hospitals in Korea.

Methods We conducted an online survey with 4,057 family medicine physicians in Korea in 2016. The results were analyzed using descriptive statistics.

Results Of the respondents, 572 doctors were working in clinics and 441 in hospitals. In the analysis of treatment pattern by doctors, the rate of chronic disease management was $84.7 \%$ in clinics and $93.4 \%$ in hospitals $(p<0.001)$, and the rate of diseases covered by national insurance was $74.8 \%$ in clinics and $76.9 \%$ in hospitals $(p=0.005)$. Among physicians younger than 40 years, the rate of chronic disease management and diseases covered by national insurance were $64.6 \%$ and $68.0 \%$ in clinics and $93.6 \%$ and $78.5 \%$ in hospitals, retrospectively.

Conclusions Family medicine physicians working in hospitals have higher rates of chronic disease management and diseases covered by national insurance. This discrepancy of treatment pattern became larger for doctors younger than 40 years. More in-depth studies of the treatment pattern and its tendencies between family medicine physicians in clinics and hospitals are needed in the future.

\section{Background}

After family medicine as practiced in the United States was introduced to the Korean medical system in 1978, the first residency program specifically for family medicine opened in 1979. The Korean Academy of Family Medicine was established in 1980 and soon became an official member of the World Organization of National Colleges, Academies and Academic Associations of General Practitioners/Family Physicians (WONCA) in 1983. Family medicine was designated as the twenty-third specialty board in Korea in 1986. As of 2016, 8,024 family medicine specialists had been certified, 135 training hospitals were established, and there were 900 family medicine residents (1). Since then, the Family Medicine Society has been working diligently in various fields to make family medicine "the center of primary care in Korea" (1). However, based on the specialization and subdivisions in Western medicine, the instability of medical delivery systems, and other political problems, Korean family physicians have expanded their practice to encompass not only comprehensive primary health care but also additional fields, including disease prevention and the promotion of alternative health services like health check-up centers, clinics providing cosmetic medical services, anti-obesity clinics, and functional medicine in areas not covered by government insurance (2).

Specialists comprise $75.9 \%$ of all Korean doctors, and and $92.6 \%$ of clinic doctors work in clinics that have diverse specialist boards. (3-5). As competition with other clinics and the absence of insurance coverage cannot ensure continuity, comprehensiveness, and family care throughout the medical system, 
the function of primary medicine as a gatekeeper has been weakened. Furthermore, patients prefer to visit secondary and tertiary medical institutions when access limitations to large hospitals are minimal. This in turn has created an unstable medical delivery system in Korea (6). Although more than thirty-five years have passed since family medicine departments were established in Korea, there have been no reports specifically about the working status of family physicians. Therefore, this study examined the current working status of family physicians and compared the working conditions of those working in clinics and hospitals.

\section{Methods}

\section{Study population}

A list of family physicians was obtained from the databases of the Korean Academy of Family Medicine $(3,141$ persons) and the Korean Society of Family Medicine (1,622 persons; Figure 1). The members' email addresses and text message contact information were provided by the database managers after confidentiality was assured. All participants provided written informed consent, and this study was approved by the Myongji Hospital Institutional Review Board (MJH-16-097).

\section{Questionnaire development and survey process}

Respondents were asked to provide basic information in the questionnaire, including their age and gender, board duration, education level (bachelor's, master's, doctorate), regional characteristics of their medical institution (large city, small-medium city, rural area), type of workplace (clinic, hospital, geriatric hospital, general hospital, or advanced general hospital), and their position in the medical institution (for clinics: owner, employee, or other; for hospitals: professor, resident, employee, or other). Two major groups were classified as either "clinic" for doctors working in clinics or "hospital" for doctors working in hospitals (geriatric, general, and advanced general). In Korea, doctors usually work in a single medical institute, thereby indicating that practices in clinics and hospitals were mutually exclusive for the purposes of this study. Chronic disease management was assessed by the following question: "Have you provided treatment for chronic diseases, such as diabetes and hypertension?" (answered yes or no). Other factors were assessed as follows: Diseases covered by national insurance by "Indicate the percentage of your patients covered by national health insurance," and patient age groups by "What proportions of your patients are younger than 18 , aged $19-64$, or older than 65 ?" For working conditions, the number of working days and working nights per week were recorded, with the standard night shift starting time established as 18:30. Online surveys were conducted over a three-month period from August 15 to December 15, 2016. Participants were regularly sent e-mails and text messages to encourage participation. 


\section{Data analysis}

Descriptive statistical analyses were performed on the results of the survey. The mean value and standard deviations of the continuous variables were calculated, as were the median and standard deviations of the categorical variables. The statistical analysis of the two groups (working in clinics and hospitals) was conducted with Student's $t$-tests and chi-square tests. Statistical significance was defined as $p<0.05$. SAS 9 statistical software (SAS Institute Inc., Cary, NC) was used for this study.

\section{Results}

From a total of 4,057 family specialists, 1,083 questionnaires were completed for a response rate of $26.7 \%$. Of these, 572 worked in clinics and 441 in hospitals (Figure 1). Table 1 shows the clinical characteristics of the family physicians in the two workplace settings. The mean age was higher in clinics than in hospitals (43.7 vs. 41.2 years old, $p<0.001$ ). The proportion of women was lower in clinics than in hospitals ( 27.6 vs. $38.6 \%, p<0.001)$, and the length of time after board certification was longer in clinics than in hospitals ( 10.8 vs. 8.6 years, $p<0.001)$. Regarding the classification of the medical institutions where the doctors provide their services, $16.6 \%$ worked in advanced general hospitals, $28.6 \%$ in general hospitals, $32.4 \%$ in geriatric hospitals, and $22.4 \%$ in hospitals. Figure 2 shows the distribution of family physicians in Korea by employment status. Doctors who were employees and/or owners of clinics comprised $34.4 \%$ and $63.5 \%$, respectively. Among the doctors working in hospitals, $74.1 \%$ were paid doctors, $13.4 \%$ professors, $8.0 \%$ residents, and $4.6 \%$ others.

Table 2 shows the medical practice characteristics provided by family physicians working in clinics and hospitals. First, the rate of chronic disease management was $84.7 \%$ in clinics and $93.4 \%$ in hospitals ( $p<$ $0.001)$. Among the physicians who were $<40$ years old, the rate of chronic disease management was $64.6 \%$ in clinics and $93.6 \%$ in hospitals $(p<0.001)$. Among those $\geq 40$ years old, the rate of chronic disease management was $93.6 \%$ in clinics and $92.7 \%$ in hospitals $(p=0.31)$. The rate of diseases covered by national insurance was $74.8 \%$ in clinics and $76.9 \%$ in hospitals $(p=0.005)$. Among the physicians $<40$ years old, the rate of diseases covered by national insurance was $68.0 \%$ in clinics and $78.5 \%$ in hospitals $(p<0.001)$. Among physicians $\geq 40$ years old, the rate of disease covered by national insurance was $81.0 \%$ in clinics and $84.7 \%$ in hospitals $(p=0.19)$. Regarding patient age, the percentages per group in clinics were the following: $<18$ (14.5\%), 19-64 (54.8\%), and $\geq 65$ (31.1\%). In hospitals, they were $<18(6.7 \%), 19-64(37.7 \%)$, and $\geq 65(55.6 \%)$, respectively $(p<0.001)$.

Table 3 shows the working conditions of family physicians measured in daily and nightly shifts per week. The mean working days per week were 5.7 for clinics and 5.3 for hospitals $(p<0.001)$, and the mean working nights per week were 3.0 for clinics and 0.8 for hospitals $(p<0.001)$. Supplementary Figure 1 presents the distribution of day shifts per week and night shifts per week. Family physicians usually worked six days per week in clinics or five days per week in hospitals, while those working night shifts usually worked five days per week. The second highest frequency was zero days per week in both clinics and hospitals. 


\section{Discussion}

In this study, we found that family physicians working in hospitals had higher rates of chronic disease management and higher rates of elderly patients compared to those working in clinics. This discrepancy increased for doctors under 40 years of age.

There are several possible reasons for the differences in medical treatment patterns in hospitals and clinics. Family physicians in Korea have expanded their range of medical services to encompass not only disease treatment but also assistance in health check-up centers, health promotion, and new advanced medical technologies, all of which are classified as medical services not currently covered by national insurance (7). As the proportion of total medical expenses classified as noncovered procedures has increased steadily in Korea, this phenomenon has also extended to the practice patterns of family physicians $(8,9)$. According to a report by the National Health Insurance Service (NHIS), noncovered medical expenses doubled between 2009 and 2014, and the proportion gradually increased from 13.4\% in 2006 to $17.1 \%$ in 2014 (10). Moreover, the number of clinicians who practice only noncovered medical areas has doubled in the last five years, including general medicine, plastic surgery, dentistry, and other fields (9). An appearance-oriented culture that emphasizes measures like anti-obesity treatments was recognized as one of the factors contributing to the demand for various cosmetic procedures, including skin care, plastic surgery, and anti-aging treatments $(11,12)$. In this survey, $44.8 \%$ of all family physicians stated that their primary reasons for selecting a noncovered medical area was economic, $20.1 \%$ mentioned personal interest, and $10.4 \%$ cited their desire to gain independence from government restrictions related to the right to medical treatment (Supplementary Table 1). A similar report regarding the operation of clinics for purely economic reasons and other considerations affecting the choice of treatment specialties was published in the Korean Medical Association's annual report, thereby indicating that this phenomenon extends beyond family physicians to other specialists who operate clinics (4).

Another reason for the evolving medical practice patterns in young family doctors is the instability of the medical delivery system that has reduced the influence of primary medical institutions throughout the medical market (13). The concept of primary care serving as a gatekeeper has yet to be established, and many challenges must be addressed before the possibility of patients having their own primary physicians becomes a reality in the Korean medical system $(14,15)$. The number of family physicians now entering the medical market has increased. Further, clinicians have historically been more vulnerable to deterioration of the medical delivery system and may have been more sensitive about facing economic hardship when choosing their primary care specialty. This is a more pressing issue for family physicians working in clinics than in hospitals (13). Therefore, we can assume that future trends regarding the types of medical treatment provided by young doctors will more likely reveal an emphasis on the provision of medical services related to the promotion of health and well-being rather than chronic disease management. There is a need for additional in-depth studies of the different tendencies of family doctors working in clinics compared to those in hospitals. 
This study also found that the amount of day and night duty was significantly higher for doctors working in clinics than in hospitals. This may be related to the operating hours of clinics and hospitals as clinics generally open later and close later than hospitals. In addition, this factor is strongly affected by the medical treatment area and practice patterns in hospitals; for example, if a family medicine department in a hospital is oriented to outpatient medical care and routine health check-up facilities, there is no need for inpatient care and night duty. Conversely, if a hospital provides mainly hospice-palliative inpatient care, this requires work by family doctors on a nightly basis. A more detailed evaluation of working hours should be performed to provide a comprehensive assessment of doctors' work environments.

The limitations of this study are as follows: First, the questionnaire survey was limited to subjective factors of the respondents, thereby leading to potential under- and over-reporting. Second, this study was conducted only with subjects who agreed to allow the use of their personal information, and there were update limitations of the group databases. Therefore, the representativeness of the sample was limited because our study was conducted with only half of the family physicians in Korea, and the actual number of respondents was insufficient. Third, the study was limited by the lack of information on population samples covering the entire range of family medicine specialists and the standardization of participants in terms of gender, age, and region. Therefore, it is possible that some results of this study may be biased because of the selection bias of family physicians who are more active in the Family Medicine Society compared to the general characteristics of the entire population of family medicine specialists in Korea. In order to overcome this limitation, large-scale studies will be necessary to conduct sophisticated research on more representative sample populations in the future. Nevertheless, to our knowledge, this study is the first to focus on family medicine specialists that helps identify the medical practices of family doctors and confirms the impact of the decisions they made during their formal training regarding the types of medical care they chose to provide.

\section{Conclusion}

More than thirty-five years have passed since family medicine was recognized as a specialized department in Korea, and consistent efforts have since been made to implement primary care medicine based on initial medical contacts, accessibility, comprehensiveness, coordination, sustainability, and accountability (14). However, there has been a lack of political support from the government for the establishment of a properly functioning primary medicine system. In fact, the role of primary clinics has been gradually reduced within a system where they must compete with hospitals. The first step to prepare for the future is to identify the current status of family physicians, in particular, how do they think and react to the competitive medical situation in Korea. With a rapidly aging society, the importance of chronic disease management and comprehensive and continuous medical care will need to be emphasized like never before. The professionals involved with family medicine as a form of primary health care provision are eager to help patients navigate Korea's challenging medical environment. This study is expected to be useful for establishing a clear direction for residency training, education programs, and for maintaining and developing the identity of family medicine. Large-scale follow-up 
studies using big data to facilitate comparisons with other medical departments will be necessary in the near future.

\section{List Of Abbreviations}

National Health Insurance Service (NHIS)

\section{Declarations}

\section{Ethics approval and consent to participate}

All participants provided written informed consent, and the study was approved by the Myongji Hospital Institutional Review Board (MJH-16-097).

\section{Consent for publication}

Not applicable

\section{Availability of data and materials}

The datasets generated and analyzed in the current study are not publicly available because of confidentiality restrictions. They are made available by the corresponding author for reasonable requests.

\section{Conflict of interest}

The authors declare that they have no competing interests.

\section{Funding}

No funding was provided for this research.

\section{Authors' contributions}

HYS made substantial contributions to the conception and design of the study, acquisition of the data, data analysis and interpretation, drafting and revising the article, submission of the final paper, and feedback management.

\section{Acknowledgments}

We would like to express our gratitude to the members of the family medicine department who cooperated with researchers and participated in the questionnaire.

\section{References}


1. The Korean academy of family medicine, homepage. http://www.kafm.or.kr/eng/. Accessed 21 August 2019.

2. Moon DH. The comparison of the primary care roles between family physicians and the other speciality doctors in private clinic, Master Dissemination, Korea university, Seoul, 2001.

3. Ministry of Health and Welfare, Health and welfare statistical year book 2017. http://www.mohw.go.kr/react/gm/sgm0601vw.jsp? PAR_MENU_ID=13\&MENU_ID=1304020201\&CONT_SEQ=292908\&page=4. Accessed 21 August 2019.

4. Korean Medical Association, Annual report membership statistics, Research institute for healthcare policy; 2014.

5. Im GJ, Choi JW, Lim SM, etc . A survey of the medical practitioners' offices in Korea

6. J Korean Med Assoc 2012; 55(4): 390-403.

7. Korea Institute for Health and Social Affairs. Issues and Improving Strategies on Korea Healthcare Delivery System. 2014-08.

8. Health insurance review \& assessment service, Costs of Health Care and Medical Care in Korea, 2018. https://www.hira.or.kr/ebooksc/ebook_474/ebook_474_201804091142360320.pdf. Accessed 21 August 2019.

8. Kang GW, The causes and resolution direction of uninsured medical expenses problems, NECA report: Gonggam NECA ; 2016.

9. Kim SH, medical institutions for $100 \%$ uninsured medical expenses doubled over the past 5 years. http://kimsh.or.kr/ps/bbs/board.php? bo_table=news_release\&wr_id=205\&sca=\&sfl=wr_subject\&stx=\%BA\%F1\%B1\%DE\%BF\%A9\&sop=and. Accessed 21 August 2019.

10. Lee JT, Kim DG, Current status and characteristics of uninsured medical expenses, KRI report focus, 2017.

11. Boo SJ, Misperception of body weight and associated factors. Nurs Health Sci 2014;16(4):468-75.

12. Jung JC. A Critical Study on Korean Women's Body Discourse. Journal of communication science. 2007;7(1):292-318.

13. Park SM, Choi EJ, Cho JJ, etc. Public Perception of the Need for Regular Family Doctors, Their Major Role, and Appropriate Training Duration. Korean J Fam Pract 2013;3:124-31.

14. Park KD, Primary Care Physician in Korea. KAFM 2002;23(6):677-87.

15. Choi YJ, Ko BS, Cho KH, etc. Concept, values, current status and prospect of primary care in Korea. J Korean Med Assoc 2013; 56(10): 856-65.

\section{Tables}


Table 1. Clinical characteristics of the family physicians working in clinics and hospitals (2016).

\begin{tabular}{|c|c|c|c|}
\hline Variables & $\begin{array}{c}\text { Clinic } \\
N=572\end{array}$ & $\begin{array}{l}\text { Hospital } \\
N=441\end{array}$ & *p value \\
\hline Age (years) & $43.7 \pm 8.7$ & $41.2 \pm 8.8$ & $<0.001$ \\
\hline Women (number/\%) & $158(27.6)$ & $170(38.6)$ & $<0.001$ \\
\hline Board duration (year) & $10.8 \pm 8.0$ & $8.6 \pm 7.9$ & $<0.001$ \\
\hline Education level (number/\%) & & & $<0.001$ \\
\hline Bachelor & $408(71.5)$ & $227(51.9)$ & \\
\hline Master & $130(22.8)$ & $128(29.3)$ & \\
\hline Doctor & $33(5.8)$ & $82(18.8)$ & \\
\hline Region of medical institution (number/\%) & & & 0.08 \\
\hline Large city & $338(59.3)$ & $230(52.9)$ & \\
\hline Small-medium city & $184(32.6)$ & $176(40.5)$ & \\
\hline Rural area & $42(7.4)$ & $29(6.7)$ & \\
\hline Classification of medical institution (number/\%) & & & $<0.001$ \\
\hline Advanced general hospital & & $73(16.6)$ & \\
\hline General hospital & & $126(28.6)$ & \\
\hline Geriatric hospital & & $143(32.4)$ & \\
\hline Hospital & & $99(22.4)$ & \\
\hline Clinic & $572(100)$ & & \\
\hline
\end{tabular}

All data are represented as mean \pm standard deviation or number (\%).

${ }^{*} p$ value from Student's $t$-test or chi-square test.

Table 2. Medical characteristics provided by family physicians working in clinics and hospitals (2016). 


\begin{tabular}{|c|c|c|c|c|}
\hline & & $\begin{array}{l}\text { Clinic } \\
N=572\end{array}$ & $\begin{array}{l}\text { Hospital } \\
N=441\end{array}$ & *p value \\
\hline Chronic disease management & & $84.7 \%$ & $93.4 \%$ & $<0.001$ \\
\hline$<40$ years old & & $64.6 \%$ & $93.6 \%$ & $<0.001$ \\
\hline$\geq 40$ years old & & $93.6 \%$ & $92.7 \%$ & 0.31 \\
\hline Disease covered by national insurance & & $74.8 \%$ & $76.9 \%$ & 0.01 \\
\hline$<40$ years old & & $68.0 \%$ & $78.5 \%$ & $<0.001$ \\
\hline$\geq 40$ years old & & $81.0 \%$ & $84.7 \%$ & 0.19 \\
\hline \multirow[t]{3}{*}{ Patients' age group in years } & $<18$ & 14.5 & 6.7 & $<0.001$ \\
\hline & $19-64$ & 54.8 & 37.7 & \\
\hline & $\geq 65$ & 31.1 & 55.6 & \\
\hline
\end{tabular}

${ }^{*} p$ value from Student's $t$-test or chi-square test.

Table 3. Working conditions of family physicians, days and nights worked per week (2016).

\begin{tabular}{cccc}
\hline Mean working days (days per week) & Clinic & Hospital & $\boldsymbol{p}$ value* \\
& & & \\
\hline Day & $5.7 \pm 0.8$ & $5.3 \pm 0.7$ & $<0.001$ \\
Night & $3.0 \pm 2.3$ & $0.8 \pm 1.3$ & $<0.001$ \\
\hline
\end{tabular}

* Student's $t$-test between clinic and hospital workers; Night shift starts from 18:30.

Supplementary Table 1. Reasons for choosing a medical field not covered by national insurance.

\begin{tabular}{lcc}
\multicolumn{1}{c}{ Total $N=422$} & $\boldsymbol{N}$ & $\boldsymbol{\%}$ \\
\hline Economic reasons & 189 & 44.8 \\
Personal interest & 85 & 20.1 \\
No special reason & 82 & 19.4 \\
Medical independence from government & 44 & 10.4 \\
Others & 22 & 5.2 \\
\hline
\end{tabular}




\section{Supplemental File Legends}

Supplementary Figure 1. Distribution of working days per week for family physicians in clinics and hospitals.

1A. Day shifts per week

1B. Night shifts per week

Figures 
Figure 1. Flow chart of study population of the present study (2016).

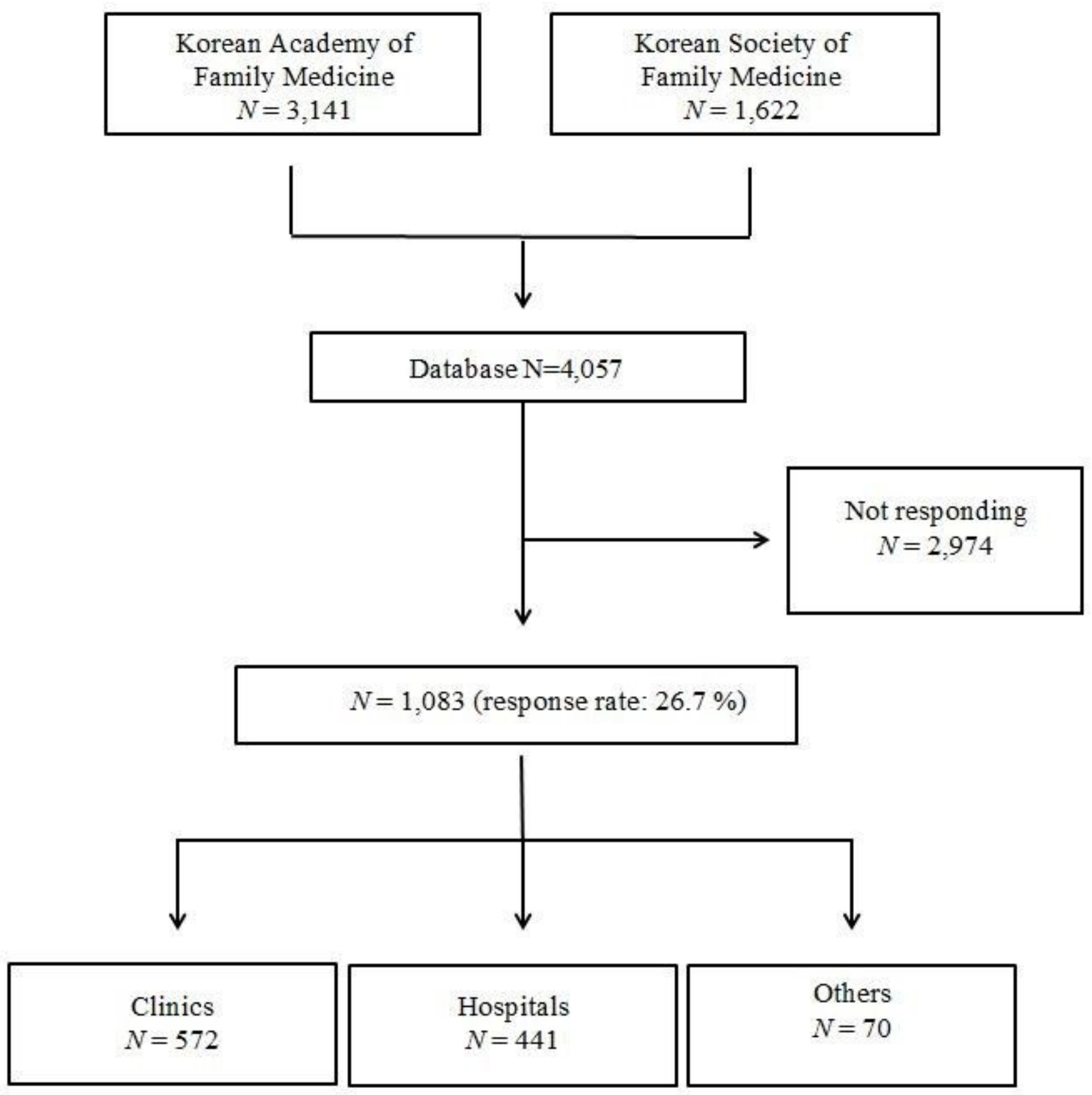

\section{Figure 1}

Flow chart of the study population (2016) 

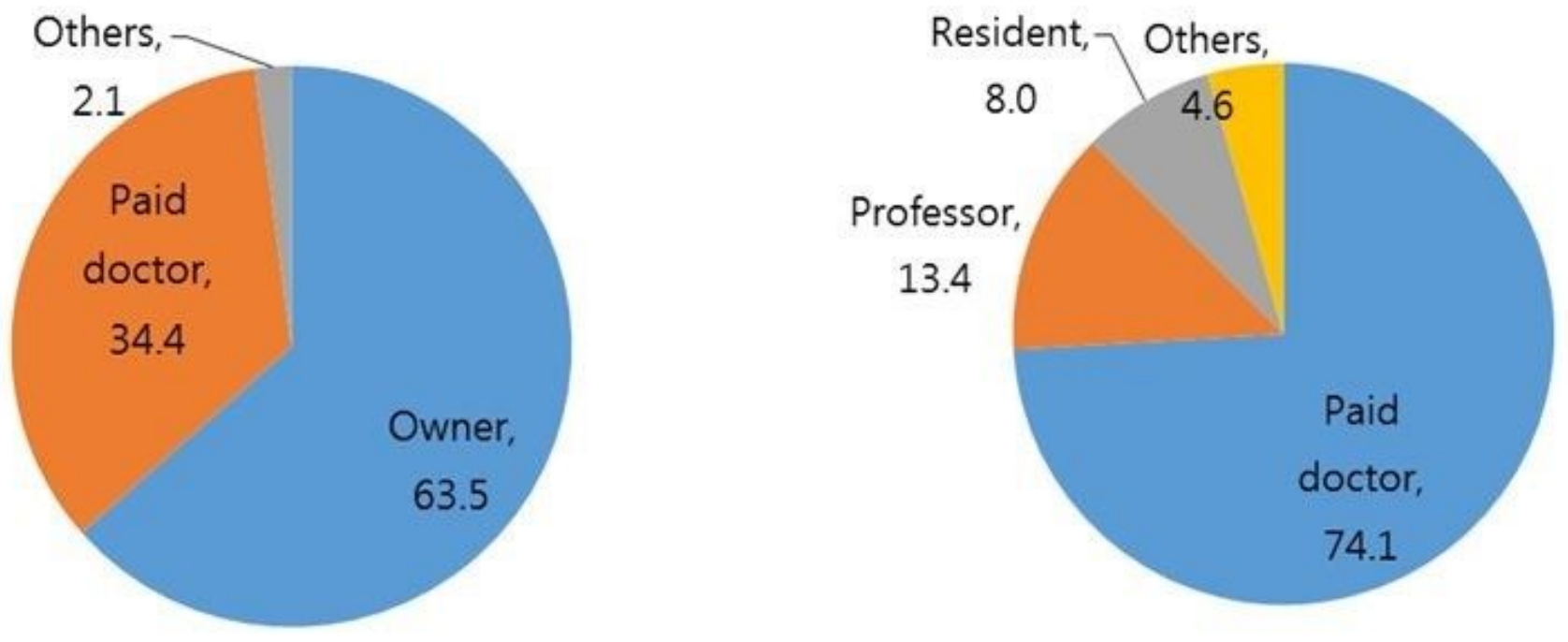

Figure 2

Distribution of family physicians in Korea by workplace type. 2A. Clinic $(N=572) 2 B$. Hospital $(N=441)$

\section{Supplementary Files}

This is a list of supplementary files associated with this preprint. Click to download.

- supplefigure12.tif

- supplefigure11.tif 